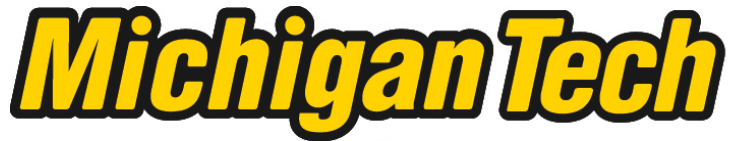 \\ Michigan Technological University Create the Future Digital Commons @ Michigan Tech
}

Computational prediction of the influence of crosslink distribution on the thermo-mechanical properties of epoxies

Ananyo Bandyopadhyay

Michigan Technological University

Follow this and additional works at: https://digitalcommons.mtu.edu/etds

Part of the Mechanical Engineering Commons

Copyright 2011 Ananyo Bandyopadhyay

\section{Recommended Citation}

Bandyopadhyay, Ananyo, "Computational prediction of the influence of crosslink distribution on the thermo-mechanical properties of epoxies", Master's report, Michigan Technological University, 2011.

https://doi.org/10.37099/mtu.dc.etds/553

Follow this and additional works at: https://digitalcommons.mtu.edu/etds

Part of the Mechanical Engineering Commons 


\title{
COMPUTATIONAL PREDICTION OF THE INFLUENCE OF CROSSLINK DISTRIBUTION ON THE THERMO-MECHANICAL PROPERTIES OF EPOXIES
}

\author{
By \\ ANANYO BANDYOPADHYAY
}

\begin{abstract}
A REPORT
Submitted in partial fulfillment of the requirements for the degree of

MASTER OF SCIENCE IN MECHANICAL ENGINEERING
\end{abstract}

MICHIGAN TECHNOLOGICAL UNIVERSITY

2011

Copyright $\odot$ Ananyo Bandyopadhyay 2011 
This report, 'Computational prediction of the influence of crosslink distribution on the thermo-mechanical properties of epoxies'

is hereby approved in partial fulfillment of the requirements for the Degree of MASTER OF SCIENCE in MECHANICAL ENGINEERING 


\section{Table of Contents}

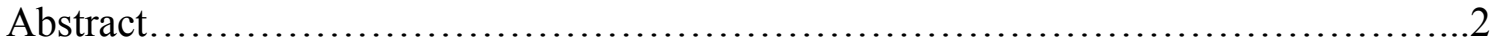

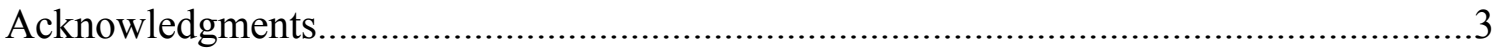

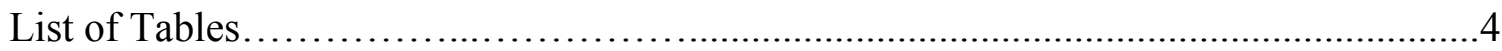

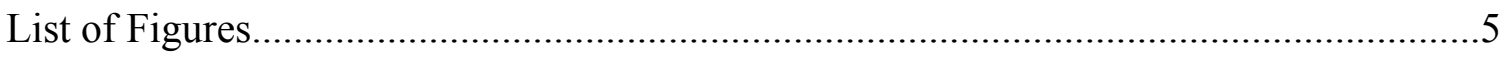

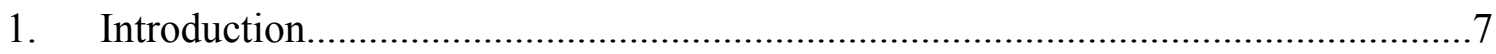

2. Molecular Modeling

2.1 Uncrosslinked molecular models..................................................................11

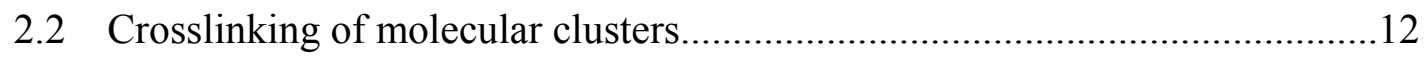

2.3 Crosslinking of large-scale molecular models..................................................13

2.4 Comparison of lengths of crosslinked bonds...................................................15

3. Results and Discussions

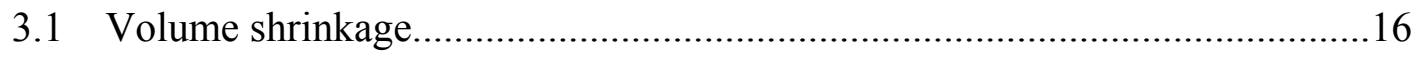

3.2 Coefficients of linear thermal expansion....................................................17

3.3 Mechanical properties................................................................................19

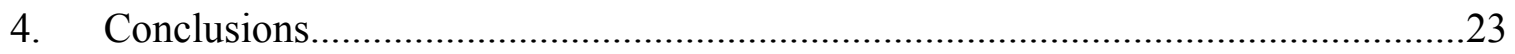

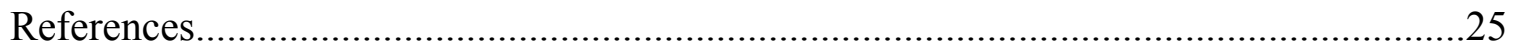

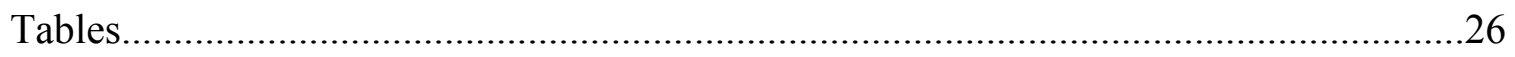

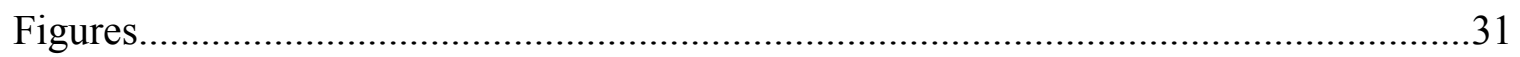




\section{Abstract}

Experimental studies on epoxies report that the microstructure consists of highly crosslinked localized regions connected with a dispersed phase of low crosslink density. The various thermo-mechanical properties of epoxies might be affected by the crosslink distribution. But as experiments cannot report the exact number of crosslinked covalent bonds present in the structure, molecular dynamics is thus being used in this work to determine the influence of crosslink distribution on thermo-mechanical properties. Molecular dynamics and molecular mechanics simulations are used to establish wellequilibrated molecular models of EPON 862-DETDA epoxy system with a range of crosslink densities and various crosslink distributions. Crosslink distributions are being varied by forming differently crosslinked localized clusters and then by forming different number of crosslinks interconnecting the clusters. Simulations are subsequently used to predict the volume shrinkage, thermal expansion coefficients, and elastic properties of each of the crosslinked systems. The results indicate that elastic properties increase with increasing levels of overall crosslink density and the thermal expansion coefficient decreases with overall crosslink density, both above and below the glass transition temperature. Elastic moduli and coefficients of linear thermal expansion values were found to be different for systems with same overall crosslink density but having different crosslink distributions, thus indicating an effect of the epoxy nanostructure on physical properties. The values of thermo-mechanical properties for all the crosslinked systems are within the range of values reported in literature. 


\section{Acknowledgments}

I am extremely thankful to Dr. Gregory M. Odegard for mentoring me in my research. While going through a few papers, Dr. Odegard and I discussed about why big ranges of values are reported from experiments on polymer materials. We thought that probably crosslinking had a significant role on these values. As experiments cannot provide a thorough understanding of crosslinking, we came up with this idea of forming models with same crosslink density but having different crosslink distributions. The entire study took four months and around 380 simulations of molecular dynamics and minimizations were done to get the results. I am grateful to Nathan Schulke and other staff of the IT department at Michigan Tech for providing the tech support. I am thankful to Dr. Ranjit Pati and Dr. Reza Shahbazian-Yassar for being in my committee. Finally, I would have never achieved my goal without the support of my wife and my parents who are back in India. I am also grateful to all the inspiration provided by my elder sister. 


\section{List of Tables}

Table 1. Summary of cluster and overall crosslink densities .26

Table 2. Different EPON 862-DETDA models with total number of crosslinks .26

Table 3. Comparison of thermal expansion (CLTE) coefficients of the nine different crosslinked systems.

Table 4. Comparison of thermal expansion coefficients of $63 \%$ crosslinked systems with different crosslinked clusters

Table 5. Comparison of thermal expansion coefficients of $70 \%$ crosslinked systems with different crosslinked clusters 28

Table 6. Comparison of thermal expansion coefficients of $76 \%$ crosslinked systems with different crosslinked clusters. 28

Table 7. Comparison of Young's moduli and Shear moduli of the nine different crosslinked systems but with same crosslink density

Table 8. Comparison of elastic moduli of $63 \%$ crosslinked systems with different crosslinked clusters. 30

Table 9. Comparison of elastic moduli of $70 \%$ crosslinked systems with different crosslinked clusters. 30

Table 10. Comparison of elastic moduli of $76 \%$ crosslinked systems with different crosslinked clusters. 30 


\section{List of Figures}

Figure 1. Molecular structures of EPON 862 resin and DETDA crosslinking molecules. Alkyl groups colored in red were considered as united atoms 31

Figure 2. Crosslinked EPON 862-DETDA system with 16:8 molecular ratio after complete equilibration. (Figure has been zoomed for easy understanding)....31

Figure 3. First step of Crosslinking reaction: The lone pair of electrons of the nitrogen atom attacks the carbon atom next to the epoxide oxygen, forming a $\mathrm{C}-\mathrm{N}$ bond and leaving a negative charge on the oxygen and a positive charge on the nitrogen. (The wavy lines represent the remaining parts of the EPON 862 and DETDA molecules in the respective structures)

Figure 4. Second and Final steps of crosslinking reaction: (A) The negatively charged oxygen abstracts a proton from the neighboring protonated amine, resulting in an alcohol group and an amine group and the crosslinking is complete. (B) The same crosslinked nitrogen reacts with another epoxide end of EPON 862 in the same way and forms two crosslinks.

Figure 5. Evolution of EPON 862-DETDA model having 432:216 stoichiometric ratio. (Atom spheres are at the same resolution for comparison of sizes of simulation boxes in the 3 figures). 33

Figure 6. Crosslinking in between clusters of EPON 862-DETDA 432:216 model. (Yellow lines indicate crosslinked bonds connecting clusters). .33

Figure 7. Frequency of crosslink formation over 0-8 Angstroms range within clusters 
Figure 8. Frequency of crosslink formation for interconnecting clusters over 0-8 Angstroms range for $70 \%$ crosslinked system..................................................34

Figure 9. Volume shrinkage curves of $54 \%, 63 \%, 70 \%$ and $76 \%$ overall crosslinked systems with $50 \%$ crosslinked clusters with respect to the volume at

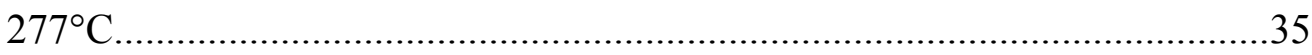

Figure 10. Volume shrinkage curves of $63 \%, 70 \%$ and $76 \%$ overall crosslinked systems with $59 \%$ crosslinked clusters with respect to the volume at $277^{\circ} \mathrm{C}$.................36

Figure 11. Volume shrinkage curves of $70 \%$ and $76 \%$ overall crosslinked systems with $69 \%$ crosslinked clusters with respect to the volume at $277^{\circ} \mathrm{C}$

Figure 12. Coefficients of linear thermal expansion vs. overall crosslink density............38

Figure 13. Coefficients of linear thermal expansion vs. R-ratio..........................................38

Figure 14. Young's and Shear moduli vs. overall crosslink density................................39

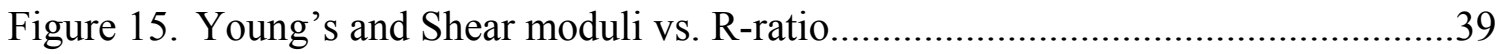




\section{INTRODUCTION}

Epoxy polymers are used as a matrix in composites for fuselages and engine casings in modern civilian and military aircraft. Because of their excellent specific stiffness and specific strength, epoxy matrix composites serve an important role in lightweight loadbearing applications. The thermo-mechanical properties of epoxy composites depend greatly on the molecular-scale structure. Because of the difficulty in experimentally determining the influence of molecular structure on small-scale thermo-mechanical behavior, computational molecular modeling is necessary for establishing accurate structure-property relationships for efficient material design and engineering.

Structural epoxy materials are formed when an epoxy monomer solution chemically combines with a hardener solution to form a crosslinked polymer network. The extent and distribution of crosslinks dictate the physical, mechanical, and thermal properties of the epoxy material. It has been shown [1-7] that the crosslink formation process does not always produce a uniformly crosslinked structure. Based on the monomer:hardener ratio, mixing conditions, temperature, pressure and time lag between curing and post-curing cycles; cured epoxies can exhibit localized regions of densely crosslinked polymer chains and other areas of loosely crosslinked chains. Vanlandingham et al. [7] studied the changes in microstructure and mechanical properties as a function of epoxy-amine mix ratio. They studied EPON 828 epoxy resin cured with PACM 20 hardener and this epoxy-amine system exhibited a two-phase structure consisting of a hard microgel phase and a dispersed phase of soft, unreacted and/or partially reacted material. Their studies showed that the size distributions in microgel regions increased with increasing amine content. Morgan and O'Neal [1] did electron and optical microscopy to study the relation between the structure and the microscopic flow and failure processes of diethylene triamine-cured bisphenol-A-diglycidyl ether epoxies. They found that these epoxies 
consisted of particles which are intramolecularly crosslinked molecular domains that can interconnect to form larger network morphological entities. The flow processes that occurred during deformation were dependent on the network morphology in which regions of either high or low crosslink density were the continuous phase. Misra et al. [5, 6] showed that typical commercial epoxy resins usually exhibit a distribution of molecular weights which result in a distribution of crosslink density in the final network. Cuthrell [2] showed that epoxy polymers contain closely packed floccules and an interstitial liquid consisting of partially reacted or unreacted material. The floccules represent highly crosslinked and entangled epoxy chains while the interstitial liquid has low crosslinked epoxy chains. Diamant et al. [3] studied the moisture absorption in differently crosslinked epoxies. They modified the epoxy networks by crosslinking with various mono/diamine proportions and found that moisture diffusion was dependant on the polymer microstructure and other factors as well. Although these studies have indicated that the crosslink distribution is not always uniform in epoxy polymers, it is difficult to experimentally quantify the exact crosslink density present at the molecular level. Infrared (IR) spectroscopy gives an approximate idea about the extent of crosslinking from the decrease of transmittance by epoxy groups with the progress of curing. As more epoxy groups crosslink by breaking the epoxide bonds, the transmittance decreases. On the other hand, the transmittance of hydroxyl groups increase with the formation of more hydroxyl bonds as a result of crosslinking. But these epoxy groups might undergo other reactions as well and hydroxyl groups might form due to other ongoing reactions. Thus the IR spectra are not always a good indicator of the extent of crosslinking. Computational methods are helpful in quantifying the exact crosslink density because prior knowledge of the number of reacting atoms and number of possible crosslinks are known. Molecular models would thus serve as an ideal tool to determine the influence of crosslink distribution on bulk-level properties. 
Many molecular dynamics (MD) studies have been performed on various epoxy systems in the last two decades. Doherty et al. [8] modeled PMA networks using latticebased simulations on a polymerization molecular dynamics scheme. Yarovsky and Evans [9] discussed a static crosslinking technique which they used to crosslink low molecularweight, water-soluble, phosphate-modified epoxy resins (CYMEL 1158). The crosslinking technique involved formation of crosslinked products in one step but all the possible crosslinks were formed in multiple steps with molecular relaxations in between. Crosslinking of epoxy resins using molecular dynamics was done by $\mathrm{Xu}$ et al. [10] and their model was used to study the diffusion of water in crosslinked networks. An iterative MD/Molecular Minimization (MM) procedure was used by them to crosslink an epoxy resin (DGEBA), with one crosslink established per iteration. Fan and Yuen [11] modeled $100 \%$ crosslinked system of 628 molecules of EPON 862-DETDA and predicted thermal and mechanical properties. Heine et al. [12] simulated large PDMS networks using a dynamic crosslinking approach and Varshney et al. [13] used Heine's dynamic crosslinking approach and Xu's MD/MM concept [10] to crosslink EPON 862 with DETDA. Varshney et al. [13] modeled systems having 14976 molecules of EPON 862 and DETDA. Although these studies demonstrated the predictive capabilities of MD for epoxy systems, they did not address the influence of crosslink distribution on the bulklevel thermo-mechanical properties.

The objective of this study is to predict the influence of crosslink distribution on the thermo-mechanical properties of an epoxy system using computational molecular modeling. Specifically, MD approaches have been used to create molecular models of EPON 862-DETDA with varying levels of overall crosslink density (i.e. total number of crosslinks formed with respect to the total number of possible crosslink reaction sites in the entire MD model) and localized "cluster" crosslink density (i.e. the crosslink density of individual regions within the entire MD model). The volume shrinkage, coefficients of thermal expansion, and elastic moduli values have been predicted for each of the various 
crosslink distributions. The results indicate that crosslink distribution may have an influence on thermal expansion coefficients and elastic moduli of the modeled epoxy.

Moreover, during commercial production, if processing conditions vary between batches of crosslinked epoxy, the distribution of crosslinks in localized regions of the crosslinked polymers can be different. This study has been done to show that though the overall extent of crosslinking might be the same in different batches of EPON 862DETDA system, the variation in distribution of localized crosslink densities can influence the thermo-mechanical properties and thus a range of values are obtained and reported instead of discrete values. 


\section{MOLECULAR MODELING}

This section describes the procedures for building a series of MD models with various distributions of crosslinks. The techniques for creating a monomer/hardener solution are described first, followed by the procedure for crosslinking individual material clusters and of the entire polymer network. It is important to note that the term "cluster" used in this study strictly refers to smaller sub-divisions of the overall MD model.

\subsection{Uncrosslinked molecular models}

A small-scale MD model was established for a mixture of EPON 862 monomer (Diglycidyl ether of Bisphenol-F) and crosslinking agent DETDA (Diethylene Toluene Diamine). The molecules of EPON-862 and DETDA are shown in Figure 1. A stoichiometric mixture (2:1 ratio) of 2 molecules of EPON-862 and 1 molecule of DETDA was modeled first using the LAMMPS (Large Scale Atomic/Molecular Massively Parallel Simulator) simulation program [14]. The initial atomic coordinates were written in a coordinate file in the native LAMMPS format and the OPLS United Atom force field [15-17] was used to define the bond, angle, and dihedral parameters with Lenard-Jones non-bonded interactions. The initial 2:1 structure was simulated in a $10 \times 10 \times 10$ angstrom $(\AA)$ simulation box with periodic boundary conditions. This structure was subjected to four energy minimizations (MM) and three MD simulations in order to drive the system to a stable equilibration. After equilibration was achieved, this structure was replicated to form eight more structures within the simulation box such that a 16:8 molecular mixture of EPON 862 and DETDA monomers was established with a total of 664 united atoms. A gradual equilibration procedure was performed over a cycle of 20 $\mathrm{MM}$ and $10 \mathrm{MD}$ simulations to equilibrate the structure and reduce the simulation box size such that a mass density of $1.2 \mathrm{~g} / \mathrm{cc}$ was achieved. All MD simulations were 
conducted in the NVT (constant volume and temperature) ensemble for 100 picoseconds at $600 \mathrm{~K}$. The NVT ensemble made use of the Nose/Hoover thermostat and barostat for temperature and pressure control, respectively [18].

\subsection{Crosslinking of molecular clusters}

After equilibration of the 16:8 molecular mixture, the structure was crosslinked based on the root mean square (RMS) distance between the $\mathrm{N}$ atoms of DETDA and $\mathrm{CH}_{2}$ groups of the EPON 862 molecules, similar to the approach used by Yarovsky and Evans [9]. A crosslinked and equilibrated 16:8 molecular mixture is shown in Figure 2 (Bonds have not been shown for better clarity of the image). The simulated crosslink process is depicted in Figures 3 and 4. Three assumptions were made for the crosslinking procedure:

1) Both primary amines in DETDA were assumed to have the same reactivity

2) The $\mathrm{CH}_{2}-\mathrm{O}$ and N-H bonds were broken simultaneously (Figure 3)

3) One $\mathrm{N}$ atom was partially activated when it had only one activated $\mathrm{CH}_{2}$ within a defined cutoff distance.

The starting point of the crosslinking reaction is shown in Figure 3 where the nucleophilic amine of the DETDA molecule reacts with the unsubstituted epoxy $\mathrm{C}$, and the adjacent oxygen atom attains a negative charge when the $\mathrm{C}-\mathrm{O}$ bond is broken. After forming a bond with the $\mathrm{C}$ atom, the $\mathrm{N}$ atom attains a positive charge and thus the neutrality of the EPON 862-DETDA system is maintained. Crosslinks were formed by computing all RMS distances between each $\mathrm{N}$ atom and the $\mathrm{CH}_{2}$ united atoms within a 
defined cutoff distance. The $\mathrm{CH}_{2}$ radicals located outside the cut-off distance of a particular $\mathrm{NH}_{2}$ group were not crosslinked to that particular group. The cutoff distance was chosen to achieve a desired level of crosslinking as described [19]. In the following step, the $\mathrm{H}$ atoms were formed by breaking $\mathrm{NH}_{2}$ bonds and were reacted with the $\mathrm{O}$ atoms of the broken epoxide ends. This bond formation was also performed based on the closest RMS distances between the $\mathrm{O}$ and $\mathrm{H}$ atoms. The second step of the crosslinking reaction is shown in Figure 4. The crosslink density of the molecular clusters is defined as the ratio of the total number of crosslinks that were formed to the 32 total crosslinks that could potentially be formed. For example, an epoxy network having 16 out of 32 crosslinks is defined as having a $50 \%$ crosslink density. A total of three molecular clusters were crosslinked to crosslink densities of $50 \%, 59 \%$, and $69 \%$.

\subsection{Crosslinking of the large-scale molecular models}

Once the molecular clusters were crosslinked and equilibrated, they were replicated to form a $3 \times 3 \times 3$ array, such that a series of larger-scale models were established that contained 27 individual molecular cluster models. Therefore, a total of three larger-scale models were initially formed, one for each of the three cluster crosslink densities $(50 \%$, $59 \%$, and 69\%). The large-scale models had 432 molecules of EPON 862 and 216 molecules of DETDA. The three large-scale systems were equilibrated in the same manner as the molecular clusters to ensure a thorough mixing of the individual molecular clusters and an overall mass density of $1.2 \mathrm{~g} / \mathrm{cc}$. A total of 17,928 united atoms were used in the model, which represented a total of 25,272 real atoms. A pictorial representation of how the large-scale structure was evolved from molecular clusters is shown in Figure 5. 
For each of the three large-scale molecular models, various levels of additional crosslinks were added to previously un-reacted reactive groups that spanned different molecular clusters within the same large-scale structure. Figure 6 shows the crosslinking of the large-scale molecular model after equilibration. The yellow lines in the figure are not actual crosslinked bonds but are a magnified representation in order to explain the presence of crosslinks. These additional crosslinks served to mechanically stabilize the structure and to achieve a series of overall crosslink densities. These crosslinks interconnecting the clusters simulated the dispersed phase of low crosslink density as indentified in the experiments which are mentioned in the introduction. The crosslinked clusters represented the regions of high crosslink density and higher molecular weight. Table 1 summarizes the nine different large-scale molecular models that were established, along with their corresponding cluster crosslink densities and overall crosslink densities. In Table 2 the different crosslinked systems having the same crosslinked clusters have been divided into columns and the exact number of crosslinks present in each system has been shown.

The ratio of the two crosslink measures is defined as

$$
R=\frac{\text { Cluster crosslink density }}{\text { Overall crosslink density }}
$$

The $R$-ratio provides a quantitative measure of the distribution of crosslinks. As shown in Table 1 , the $R$-ratio varies from 0.66 to 0.99 for the large scale models. Larger values of $R$ indicate that a higher percentage of the crosslinks are confined to the clusters, whereas small values indicate that the crosslinks are more distributed between clusters. It is also important to note that there is one 54\% overall crosslinked system, two $63 \%$ overall crosslinked systems, three $70 \%$ overall crosslinked systems, and three $76 \%$ overall crosslinked systems. Therefore, the predicted properties can be compared in terms of cluster crosslink density, overall crosslink density, and $R$-ratio. 


\subsection{Comparison of lengths of crosslinked bonds}

Bond lengths of crosslinks formed inside clusters were examined and a plot of the frequency of bonds formed within 0-8 Angstroms at intervals of 0.5 Angstrom is shown in Figure 7. As mentioned above, the crosslinks were formed based on the RMS distances between $\mathrm{N}$ and $\mathrm{CH} 2$ atoms and also between $\mathrm{O}$ and $\mathrm{H}$ atoms. Figure 7 shows that for $50 \%, 59 \%$ and $69 \%$ crosslinked clusters, the majority of crosslinks were formed in the range of 2-4.5 Angstroms. The crosslinks started forming inside the clusters when RMS distances were in the range of 0.5-1 Angstrom. Figure 8 shows the number of crosslinks formed by inter connecting the clusters in the $70 \%$ crosslinked model after the 27 clusters were brought close enough to get an equilibrated model. It is evident that most of the crosslinks were formed in the range of 2.5-5.5 Angstroms. Identical trends were seen for $63 \%$ and $76 \%$ crosslink systems and thus the plots have not been shown here. Based on the modeling procedures used over here, Figures 7 and 8 show that crosslinking in EPON 862-DETDA system occurs rapidly when the crosslinking atoms are in the range of 2-6 Angstroms. Based on the modeling procedures and force field used in this work, it is observed that the crosslinking $\mathrm{C}$ and $\mathrm{N}$ atoms prefer to be at a distance of 2-6 Angstroms in the equilibrated system. 


\section{Results and Discussions}

\subsection{Volume shrinkage}

For each of the nine cross-linked epoxy models, NPT (constant pressure and constant temperature) simulations were run for 400 picoseconds at 29 different temperatures ranging from $-73^{\circ} \mathrm{C}(200 \mathrm{~K})$ to $277^{\circ} \mathrm{C}(550 \mathrm{~K})$ at temperature intervals of $12.5^{\circ} \mathrm{C}$ and at pressures of 1 atmosphere (atm). These simulations modeled the process of constant heating of the epoxy systems from cryogenic to elevated temperatures, and provided the equilibrated volumes corresponding to each temperature interval. Shown in Figure 9 is the volume shrinkage of the four large-scale models with a 50\% cluster crosslink density (systems 1-4 in Table 1) with respect to the volume at $277^{\circ} \mathrm{C}$. Similarly, the volume shrinkage curves of the three large-scale models with a $59 \%$ cluster crosslink density (systems 5-7 in Table 1) are shown in Figure 10, and the volume shrinkage curves of the two large-scale models with a 69\% cluster crosslink density (systems 8 and 9 in Table 1) are shown in Figure 11. Data from the final 350 picoseconds of each NPT simulation were used to establish the data points in the graphs. The data gathered during the first 50 picoseconds of the simulations were not used for plotting because molecular structures typically undergo rapid relaxation during the initial stages of MD simulations.

The data in Figures 9-11 shows that for decreasing simulation temperatures, the volume shrinkage increases, as expected, due to thermal contraction. The data also clearly shows that for increasing levels of overall crosslink density the volume shrinkage decreases. This trend holds for all three cluster crosslink densities, and is similar to that reported by Bandyopadhyay et al. [19]. 
The epoxy systems shown in Figure 9 are different in one aspect; the number of crosslinks in between the crosslinked clusters. As these crosslinks connecting the clusters are considered as the dispersed phase of low crosslink density, Figure 9 shows that the clusters become more rigidly bonded to each other as the dispersed phase becomes more crosslinked. Thus the entire system becomes more resistant to volumetric deformations for a given temperature change. These results are expected, and are presented here as a validation of the present modeling technique.

\subsection{Coefficients of linear thermal expansion}

Linear regression lines were fitted on the first 10 data points $\left(-73\right.$ to $\left.39.5^{\circ} \mathrm{C}\right)$ and the last 10 data points $\left(164.5\right.$ to $\left.277^{\circ} \mathrm{C}\right)$ of the volume shrinkage curves shown in Figures 9, 10 and 11 for determining the coefficients of volumetric thermal expansion (CVTE) below and above $T_{g}$, respectively. The $T_{g}$ of similar EPON-DETDA systems have been reported elsewhere. $[11,13,20]$ The remaining 9 data points in the middle portion of the volume shrinkage curves were assumed to be the glass transition range and were not used in calculating CVTE. The CVTE is defined as

$$
\beta=\frac{1}{V_{0}}\left(\frac{\partial V}{\partial T}\right)_{P}
$$

where $V_{0}$ is the initial volume of the simulation box at $277^{\circ} \mathrm{C}$, and the subscript $P$ implies a constant-pressure process. The coefficient of linear thermal expansion (CLTE) is defined as

$$
\alpha=\frac{\beta}{3}=\frac{1}{L_{0}}\left(\frac{\partial L}{\partial T}\right)_{P}
$$


where $L_{0}$ is the initial length of each side of the cubic simulation box at $277^{\circ} \mathrm{C}$. The CLTE values obtained for the nine different crosslinked systems are shown in Figure 12. These values are also tabulated and shown in Table 3 as well.

The predictions shown in Figure 12 are consistent with those described in the literature $[11,19,21]$, that is, the CLTE values decrease with increasing levels of overall crosslink density, both above and below $\mathrm{T}_{\mathrm{g}}$. This is likely because of increasing numbers of covalent crosslinks in the polymer network serve to inhibit volume expansion in response to temperature increases. The larger values of CLTE above $T_{g}$ are due to the increase in molecular mobility of the polymer network in the rubbery state. In the glassy state, the network has a higher density and thus reduced molecular mobility decreases the material's volumetric response to temperature changes. This data demonstrates the level of scatter that is expected when determining thermo-mechanical properties of molecular models of this size. Because the expected trend in CLTE is predicted, this data also serves to validate the current molecular modeling approach.

Comparison of CLTE values between the systems of same overall crosslink density are shown in Tables 4, 5 and 6. Table 4 shows the comparison of CLTE values of two $63 \%$ crosslinked systems but with differently crosslinked clusters. The comparison of systems with same overall crosslink density but differently crosslinked clusters is analogous to the comparison among systems having the same degree of cure but with different crosslink distributions in their microstructures. Table 5 shows the comparison among three $70 \%$ crosslinked systems and Table 6 shows the comparison among three $76 \%$ crosslinked systems with varying crosslink distributions. Each of the three tables shows variation in values of CLTE above and below $T_{g}$ because of crosslink distributions. Though there is no apparent trend in the values with change in cluster crosslink density, the variation itself is a good indication of the influence of nanostructural changes on the thermal properties. 
The CLTE data is plotted against the R-ratio in Figure 13. Above $T_{g}$, there is a considerable amount of scatter in the data, and there is no clear influence of R-ratio on the CLTE. Below $\mathrm{T}_{\mathrm{g}}$, the scatter in the data is reduced and it appears that there may be a slight increase in the CLTE for increases in R-ratio. This would indicate that as more crosslinks are confined to the individual clusters, the overall molecular mobility of the system increases, as there is less resistance to volumetric changes with respect to temperature changes.

\subsection{Mechanical properties}

The elastic constants of the nine crosslinked systems were predicted with MD simulations. Uniaxial tensile strains and volumetric strains were applied to the MD models at different deformation magnitudes to establish the elastic properties. All simulations were performed in the NVT ensemble under periodic boundary conditions. For all simulations, the applied strains were ramped up to the final strain level over the duration of the simulated time. The virial stress components were calculated for each timestep over the entire loading range. It was assumed that the bulk material mechanical response was linear-elastic and isotropic. It is important to note that although the elastic response of polymers is generally dependent on applied strain rate, and the simulated deformations are at relatively high strain rates, it is expected that the predicted elastic properties are close to those observed experimentally.

For the volumetric deformations, equal axial strains were applied simultaneously over all three principal directions of the simulation boxes. The simulations were run at $300 \mathrm{~K}$ (room temperature) for 200 picoseconds with timesteps of 0.2 femtoseconds. The simulated deformations were applied in both tension and compression according to 


$$
\varepsilon_{x x}=\varepsilon_{y y}=\varepsilon_{z z}= \pm 0.003, \pm 0.005, \pm 0.007
$$

where $\varepsilon_{x x}, \varepsilon_{y y}$, and $\varepsilon_{z z}$ are the axial components of the infinitesimal strain tensor component with respect to the basis vectors defined in Figure 5. The overall dilatation of the molecular models was

$$
\Delta=\varepsilon_{x x}+\varepsilon_{y y}+\varepsilon_{z z}
$$

For each timestep in these simulations, the overall hydrostatic stress $\sigma_{h}$ of the model was calculated by

$$
\sigma_{h}=\frac{1}{3}\left(\sigma_{x x}+\sigma_{y y}+\sigma_{z z}\right)
$$

where $\sigma_{x x}, \sigma_{y y}$, and $\sigma_{z z}$ are the volume-averaged stress tensor components. For each timestep of the final 180 picoseconds of the simulations, the hydrostatic stress and dilatation were used to perform a linear regression analysis to determine the bulk modulus

$$
K=\frac{\sigma_{h}}{\Delta}
$$

The bulk moduli calculated for positive and negative dilatations over each of the three strain levels were averaged. The data gathered during the first 20 picoseconds of the simulations were not used in the regression analysis because molecular structures typically undergo rapid relaxation during the initial stages of MD simulation. The structures were well-equilibrated before the start of these deformations and thus did not need significant time for relaxation. In the initial 20 picoseconds of the deformation simulation, the initial oscillation of the total pressure values dissipated. Nearly one million data points were used to calculate the bulk modulus for each epoxy system. 
For the uniaxial tensile deformations, strains were individually applied along each of the principal axes of the simulation box. Axial strains were applied such that Poisson contractions were allowed in the transverse directions. For each model, the tensile strain was divided equally over 200 picoseconds with timesteps of 0.4 femtoseconds. Tensile strains of magnitudes $\pm 0.003, \pm 0.005$, and \pm 0.007 were applied in each direction.

A linear regression analysis was performed over the final 180 picoseconds of each simulation to determine the corresponding Young's modulus $(E)$. The Young's moduli were averaged over all three axes and all three strain levels in both tension and compression.

Using the values obtained for Bulk moduli and Young's moduli, Shear moduli (G) were calculated for all nine molecular systems as

$$
G=\frac{3 K E}{9 K-E}
$$

The predicted Young's and shear moduli are shown in Figure 14 for the entire range of overall crosslink densities. From the figure it is clear that there is an increasing trend in both moduli as the crosslink density increases. The magnitude of the predicted elastic properties is similar to those reported in the literature for the same epoxy system. This trend with respect to crosslink density is similar to that observed by Bandyopadhyay et al [19] for the same epoxy system. Similar increasing trends have been reported in other epoxy systems by Gupta et al. [22] and in epoxy-nanotube composites by Miyagawa et al. [23]. Lees and Davidson [24] discuss data that show increasing distances between reactive sites in epoxies result in decreasing crosslink densities and decreasing elastic properties. Figure 14 also indicates that there is a substantial amount of scatter in the predicted elastic properties over the range of overall crosslink densities, particularly at overall crosslink densities of $63 \%$ and $70 \%$. The reason for this scatter becomes clear by 
looking at the comparison of elastic moduli values of systems with same overall crosslink density as shown in Tables 8, 9 and 10. Table 7 shows the elastic moduli of the nine crosslinked systems.

Table 8 shows that Young's modulus varies from $0.79 \mathrm{GPa}$ to $1.32 \mathrm{GPa}$ while shear modulus varies from 0.27 to 0.46 when the crosslink distributions change in the two systems with $63 \%$ overall crosslink density. Similarly, changes in moduli are observed in the three systems with $70 \%$ overall crosslink density but differently crosslinked clusters as shown in Table 9 . While systems with $76 \%$ crosslink density show variation in moduli in Table 10, the variation was not found to be large. Similar thing is noticed in Figure 14 where these same moduli values are plotted at $76 \%$ overall crosslink density. This observation is important because it shows that at high crosslink densities, the network becomes highly entangled and the distribution of crosslinks does not influence the mechanical properties anymore. So there is a limiting range of high crosslink density, where the crosslinks inside and outside the clusters make the model rigid enough to have no influence of crosslink distribution on them.

Figure 15 shows the Young's and shear moduli plotted as a function of R-ratio. The regression lines show R-ratio has no influence on moduli values. R-ratio changes as crosslinked bonds become more frequent inside the clusters than outside and vice-versa. As there is an addition of bonds inside clusters and a subtraction of bonds outside clusters for an increasing R-ratio, there is a dual effect of crosslinked bonds on the moduli. It maybe for this dual effect, that no change in moduli values were seen in Figure 15 but the influence of crosslink distribution on elastic moduli is evident from the Tables 8,9 and 10. 


\section{Conclusions}

A molecular modeling study using molecular dynamics and minimizations has been done on EPON 862-DETDA polymer. Crosslinked systems with varying degrees of total number of crosslinks and varying degrees of crosslinks inside localized clusters have been modeled. Thermal expansion coefficients and elastic properties of the epoxy system were predicted for these crosslinked systems. The predicted thermo-mechanical properties were mostly consistent with those given in literature, thus validating the computational approach used. The results indicate that with increasing crosslink density the elastic stiffness tends to increase, while the coefficient of thermal expansion decreases, both above and below $\mathrm{T}_{\mathrm{g}}$. The results also indicate that variation in crosslink distribution leads to microstructural changes which affect thermal and mechanical properties.

Volume shrinkage curves were different for systems having the same crosslink density but with differently crosslinked localized regions or clusters. The coefficients of thermal expansion for systems with different crosslink distributions were different indicating the influence of crosslink distribution. For coefficient of linear expansion, R-ratio had a definite influence below $\mathrm{T}_{\mathrm{g}}$. The CLTE increased with increase in R-ratio which indicated that as crosslinks are confined to clusters, molecular mobility increases.

Young's modulus and shear modulus for the above mentioned systems have been reported in this work. Both moduli had increasing trends with increase in overall crosslink density. The moduli values also changed among the different systems due to changes in crosslink distribution. The systems with the highest overall crosslink density but varying crosslink distributions had a highly entangled nanostructure which had little influence of crosslink distribution. Linear regression lines for data of both moduli showed 
that there was no dependence on R-ratio. The important conclusion of this work is that an in-depth computational study of the crosslink distribution validates experimental findings about the coexistence of small regions of high crosslinked density and a dispersed phase of low crosslink density. This work also shows the influence of crosslink distribution on the thermo-mechanical properties of the cured epoxy systems. As it is impossible to know the exact crosslink density of epoxy systems experimentally, this study is important in knowing the influence of crosslink distribution in a quantitative way. . 


\section{References}

1. Morgan RJ and Oneal JE. Journal of Materials Science 1977;12(10):1966-1980.

2. Cuthrell RE. Journal of Applied Polymer Science 1963;12(6):1263-1278.

3. Diamant Y, Marom G, and Broutman LJ. Journal of Applied Polymer Science 1981;26(9):3015-3025.

4. S.S. Labana SN, A.J. Chompff. In Polymer Networks: Structure and Mechanical Properties. New York, 1971. pp. 453.

5. Misra SC, Manson JA, and Sperling LH. Abstracts of Papers of the American Chemical Society 1978;176(Sep):32-32.

6. Misra SC, Manson JA, and Sperling LH. Abstracts of Papers of the American Chemical Society 1978;176(Sep):33-33.

7. Vanlandingham MR, Eduljee RF, and Gillespie JW. Journal of Applied Polymer Science 1999;71(5):699-712.

8. Doherty DC, Holmes BN, Leung P, and Ross RB. Computational and Theoretical Polymer Science 1998;8(1-2):169-178.

9. $\quad$ Yarovsky I and Evans E. Polymer 2002;43(3):963-969.

10. Wu CF and Xu WJ. Polymer 2006;47(16):6004-6009.

11. Fan HB and Yuen MMF. Polymer 2007;48(7):2174-2178.

12. Heine DR, Grest GS, Lorenz CD, Tsige M, and Stevens MJ. Macromolecules 2004;37(10):3857-3864.

13. Varshney V, Patnaik SS, Roy AK, and Farmer BL. Macromolecules 2008;41(18):68376842.

14. Plimpton S. Journal of Computational Physics 1995;117(1):1-19.

15. Jorgensen WL, Maxwell DS, and TiradoRives J. Journal of the American Chemical Society 1996;118(45):11225-11236.

16. Weiner SJ, Kollman PA, Case DA, Singh UC, Ghio C, Alagona G, Profeta S, and Weiner P. Journal of the American Chemical Society 1984;106(3):765-784.

17. Watkins EK and Jorgensen WL. Journal of Physical Chemistry A 2001;105(16):41184125 .

18. Hoover WG. Physical Review A 1985;31(3):1695-1697.

19. Bandyopadhyay A, Valavala PK, Clancy TC, Wise KE, and Odegard GM. Polymer (in press) 2011.

20. Miller S. RGD, Bail J.L., Kohlman L.W., Binienda W.K. . Effects of Hygrothermal Cycling on the Chemical, Thermal, and Mechanical Properties of 862/W Epoxy Resin. Aircraft Airworthiness and Sustainment Conference. Austin, TX, 2010.

21. Wang SR, Liang ZY, Gonnet P, Liao YH, Wang B, and Zhang C. Advanced Functional Materials 2007;17(1):87-92.

22. Gupta VB, Drzal LT, Lee CYC, and Rich MJ. Polymer Engineering and Science 1985;25(13):812-823.

23. Miyagawa H and Drzal LT. Polymer 2004;45(15):5163-5170.

24. Lees S and Davidson CL. Journal of Biomechanics 1977;10(8):473-486. 


\section{Tables}

Table 1. Summary of cluster and overall crosslink densities

\begin{tabular}{|c|c|c|c|}
\hline $\begin{array}{c}\text { Large-scale } \\
\text { molecular model }\end{array}$ & $\begin{array}{c}\text { Cluster } \\
\text { crosslink density }\end{array}$ & $\begin{array}{c}\text { Overall } \\
\text { crosslink density }\end{array}$ & R-ratio \\
\hline $\mathbf{1}$ & $50 \%$ & $54 \%$ & 0.93 \\
\hline $\mathbf{2}$ & $50 \%$ & $63 \%$ & 0.79 \\
\hline $\mathbf{3}$ & $50 \%$ & $70 \%$ & 0.72 \\
\hline $\mathbf{4}$ & $50 \%$ & $76 \%$ & 0.66 \\
\hline $\mathbf{5}$ & $59 \%$ & $63 \%$ & 0.94 \\
\hline $\mathbf{6}$ & $59 \%$ & $70 \%$ & 0.85 \\
\hline $\mathbf{7}$ & $59 \%$ & $76 \%$ & 0.78 \\
\hline $\mathbf{8}$ & $69 \%$ & $70 \%$ & 0.98 \\
\hline $\mathbf{9}$ & $69 \%$ & $76 \%$ & 0.91 \\
\hline
\end{tabular}

Table 2. Different EPON 862-DETDA models with total number of crosslinks

\begin{tabular}{|c|c|c|c|}
\hline $\begin{array}{c}\text { Formed } \\
\text { crosslinks/Total } \\
\begin{array}{c}\text { number of possible } \\
\text { crosslinks }\end{array}\end{array}$ & $\begin{array}{c}1^{\text {st }} \text { batch of models } \\
\text { with } 50 \% \\
\text { crosslinked } 16: 8 \\
\text { entanglements }\end{array}$ & $\begin{array}{c}2^{\text {nd }} \text { batch of } \\
\text { models with } 59 \% \\
\text { crosslinked } 16: 8 \\
\text { entanglements }\end{array}$ & $\begin{array}{c}3^{\text {rd }} \text { batch of models } \\
\text { with } 69 \% \\
\text { crosslinked } 16: 8 \\
\text { entanglements }\end{array}$ \\
\hline $466 / 864$ & $54 \%$ & \multicolumn{2}{|c|}{} \\
\hline $544 / 864$ & $63 \%$ & $63 \%$ & \multicolumn{2}{|c|}{$70 \%$} \\
\hline $604 / 864$ & $70 \%$ & $70 \%$ & $76 \%$ \\
\hline $656 / 864$ & $76 \%$ & $76 \%$ & \multicolumn{2}{|c|}{} \\
\hline
\end{tabular}


Table 3. Comparison of thermal expansion coefficients of the nine different crosslinked systems

\begin{tabular}{|c|c|c|c|c|}
\hline \multicolumn{5}{|c|}{ Coefficients of Linear Thermal Expansion $(\alpha) \times 10^{-5}$} \\
\hline \multicolumn{5}{|c|}{ Crosslinked systems with $50 \%$ locally crosslinked clusters } \\
\hline & $54 \%$ & $63 \%$ & $70 \%$ & $76 \%$ \\
\hline Above $\mathbf{T}_{\mathrm{g}}$ & 22.7 & 21.7 & 18.3 & 17.3 \\
\hline Below $\mathbf{T}_{g}$ & 10.3 & 9.0 & 8.0 & 7.0 \\
\hline \multicolumn{5}{|c|}{ Crosslinked systems with $59 \%$ locally crosslinked clusters } \\
\hline & \multicolumn{2}{|c|}{$63 \%$} & $70 \%$ & $76 \%$ \\
\hline Above $\mathbf{T}_{\mathrm{g}}$ & \multicolumn{2}{|c|}{15.3} & 13.3 & 11.7 \\
\hline Below $\mathbf{T}_{\mathrm{g}}$ & \multicolumn{2}{|c|}{8.0} & 7.7 & 5.7 \\
\hline \multicolumn{5}{|c|}{ Crosslinked systems with $69 \%$ locally crosslinked clusters } \\
\hline & \multicolumn{2}{|c|}{$70 \%$} & \multicolumn{2}{|c|}{$76 \%$} \\
\hline Above $\mathbf{T}_{\mathrm{g}}$ & \multicolumn{2}{|c|}{16.7} & \multicolumn{2}{|c|}{14.3} \\
\hline Below $\mathbf{T}_{g}$ & \multicolumn{2}{|c|}{8.3} & \multicolumn{2}{|c|}{7.7} \\
\hline
\end{tabular}


Table 4. Comparison of thermal expansion coefficients of $63 \%$ crosslinked systems with different crosslinked clusters

\begin{tabular}{|c|c|c|}
\hline \multicolumn{2}{|c|}{ CLTE of systems with $\begin{array}{c}\text { 63\% overall crosslinking but different crosslink } \\
\text { distributions }\end{array}$} \\
\hline & From $\mathbf{5 0 \%}$ cluster & From $\mathbf{5 9 \%}$ cluster \\
\hline Above Tg & 21.7 & 15.3 \\
\hline Below Tg & 9.0 & 8.0 \\
\hline
\end{tabular}

Table 5. Comparison of thermal expansion coefficients of $70 \%$ crosslinked systems with different crosslinked clusters

\begin{tabular}{|c|c|c|c|}
\hline \multicolumn{3}{|c|}{ CLTE of systems with 70\% overall crosslinking but different crosslink } \\
distributions \\
\hline & From $\mathbf{5 0 \%}$ cluster & From 59\% cluster & From $\mathbf{6 9 \%}$ cluster \\
\hline Above Tg & 18.3 & 13.3 & 16.7 \\
\hline Below Tg & 8.0 & 7.7 & 8.3 \\
\hline
\end{tabular}

Table 6. Comparison of thermal expansion coefficients of $76 \%$ crosslinked systems with different crosslinked clusters

\begin{tabular}{|c|c|c|c|}
\hline \multicolumn{3}{|c|}{ CLTE of systems with $\begin{array}{c}\mathbf{7 6 \%} \text { overall crosslinking but different crosslink } \\
\text { distributions }\end{array}$} \\
\hline & From $\mathbf{5 0 \%}$ cluster & From 59\% cluster & From 69\% cluster \\
\hline Above Tg & 17.3 & 11.7 & 14.3 \\
\hline Below Tg & 7.0 & 5.7 & 7.7 \\
\hline
\end{tabular}


Table 7. Comparison of Young's moduli and Shear moduli of the nine different crosslinked systems

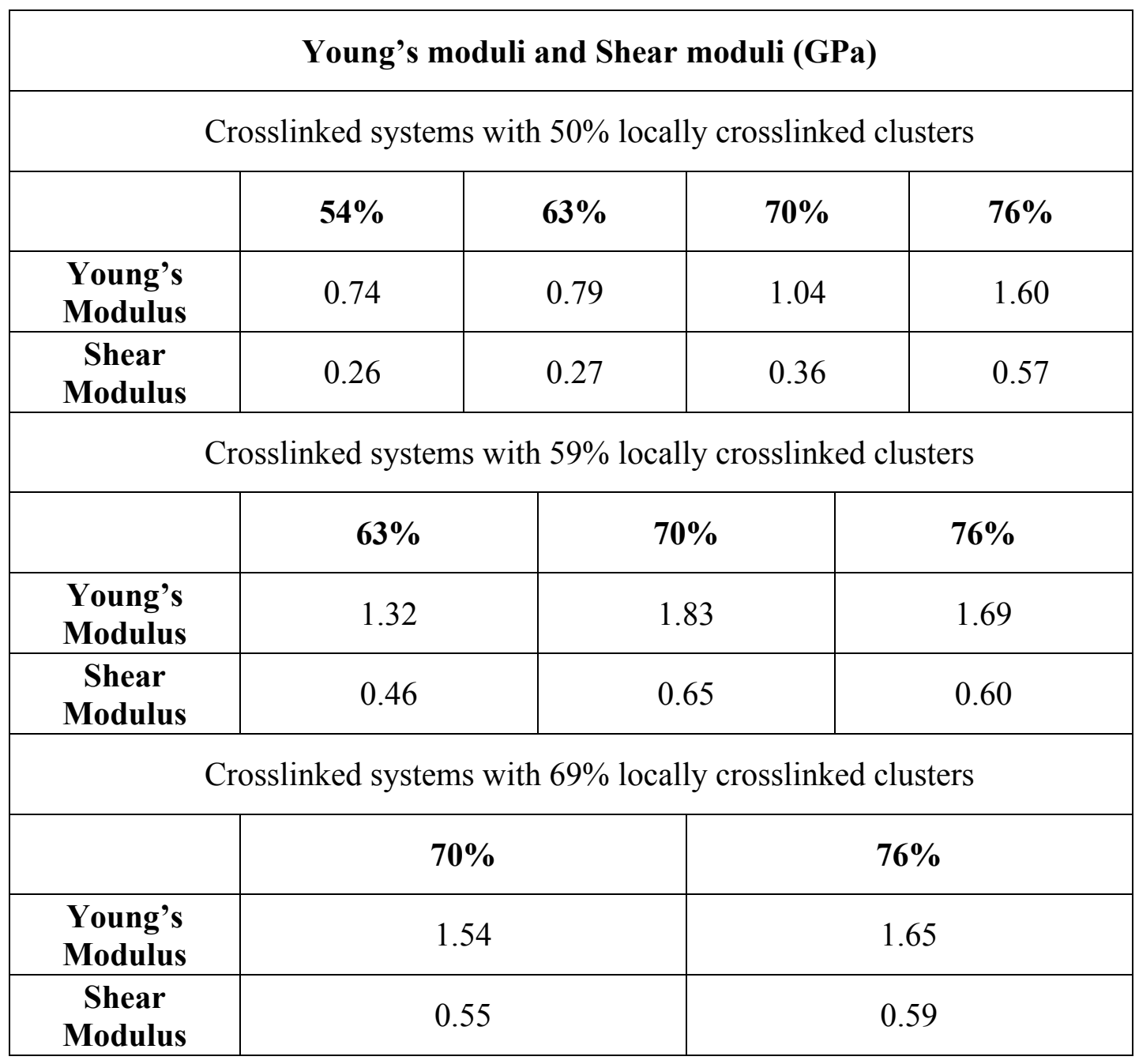


Table 8. Comparison of elastic moduli of $63 \%$ crosslinked systems with different crosslinked clusters

\begin{tabular}{|c|c|c|}
\hline \multicolumn{3}{|c|}{$\begin{array}{c}\text { Elastic moduli of systems with 63\% overall crosslinking but different } \\
\text { crosslink distributions (values in GPa) }\end{array}$} \\
\hline & From 50\% cluster & From 59\% cluster \\
\hline Young's Modulus & 0.79 & 1.32 \\
\hline Shear Modulus & 0.27 & 0.46 \\
\hline
\end{tabular}

Table 9. Comparison of elastic moduli of $70 \%$ crosslinked systems with different crosslinked clusters

\begin{tabular}{|c|c|c|c|}
\hline \multicolumn{3}{|c|}{ Elastic moduli of systems with 70\% overall crosslinking but different } \\
crosslink distributions (values in GPa)
\end{tabular}

Table 10 . Comparison of elastic moduli of $76 \%$ crosslinked systems with different crosslinked clusters

\begin{tabular}{|c|c|c|c|}
\hline \multicolumn{3}{|c|}{ Elastic moduli of systems with $\begin{array}{r}\mathbf{7 6 \%} \text { overall crosslinking but different } \\
\text { crosslink distributions (values in GPa) }\end{array}$} \\
\hline & From $\mathbf{5 0 \%}$ cluster & From 59\% cluster & From $\mathbf{6 9 \%}$ cluster \\
\hline $\begin{array}{c}\text { Young's } \\
\text { Modulus }\end{array}$ & 1.60 & 1.69 & 1.65 \\
\hline $\begin{array}{c}\text { Shear } \\
\text { Modulus }\end{array}$ & 0.57 & 0.60 & 0.59 \\
\hline
\end{tabular}




\section{Figures}<smiles>CCc1cc(CC)c(N)c(C)c1N</smiles>

Figure 1. Molecular structures of EPON 862 resin and DETDA crosslinking molecules. Alkyl groups colored in red were considered as united atoms.

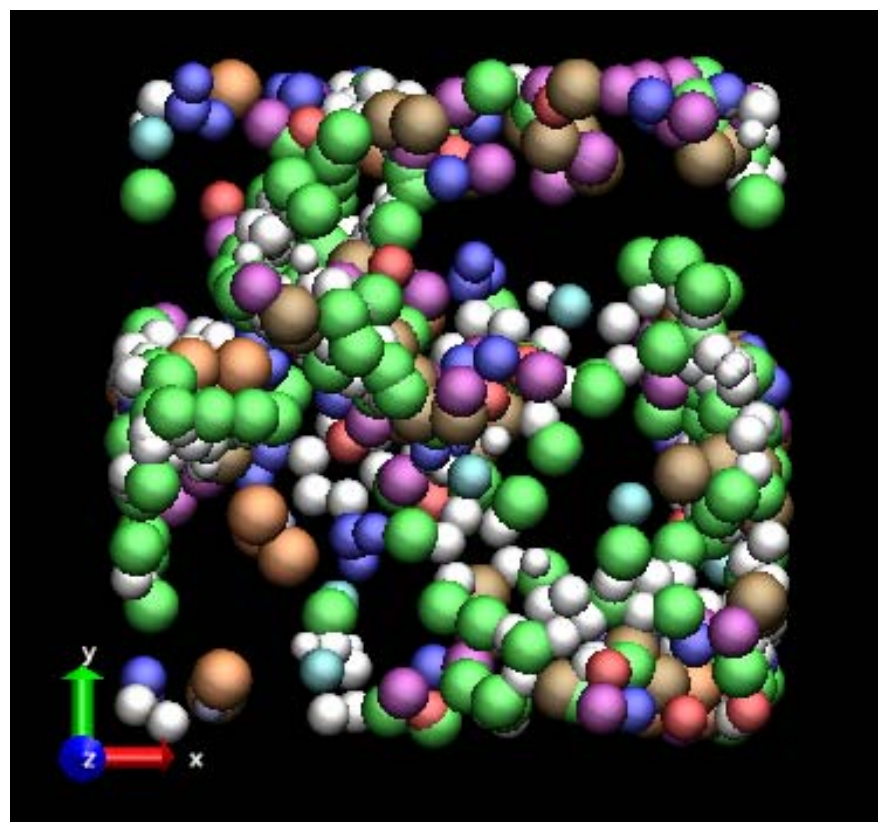

Figure 2. Crosslinked EPON 862-DETDA system with 16:8 molecular ratio after complete equilibration. (Figure has been zoomed for easy understanding) 


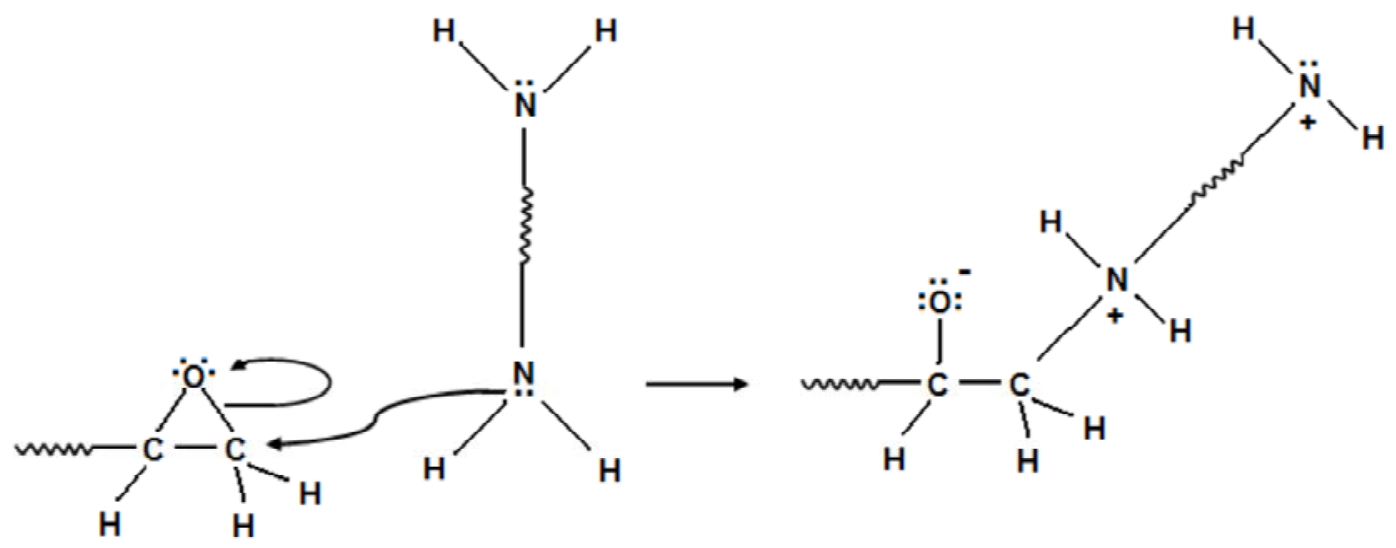

Figure 3. First step of Crosslinking reaction: The lone pair of electrons of the nitrogen atom attacks the carbon atom next to the epoxide oxygen, forming a $\mathrm{C}-\mathrm{N}$ bond and leaving a negative charge on the oxygen and a positive charge on the nitrogen. (The wavy lines represent the remaining parts of the EPON 862 and DETDA molecules in the respective structures)
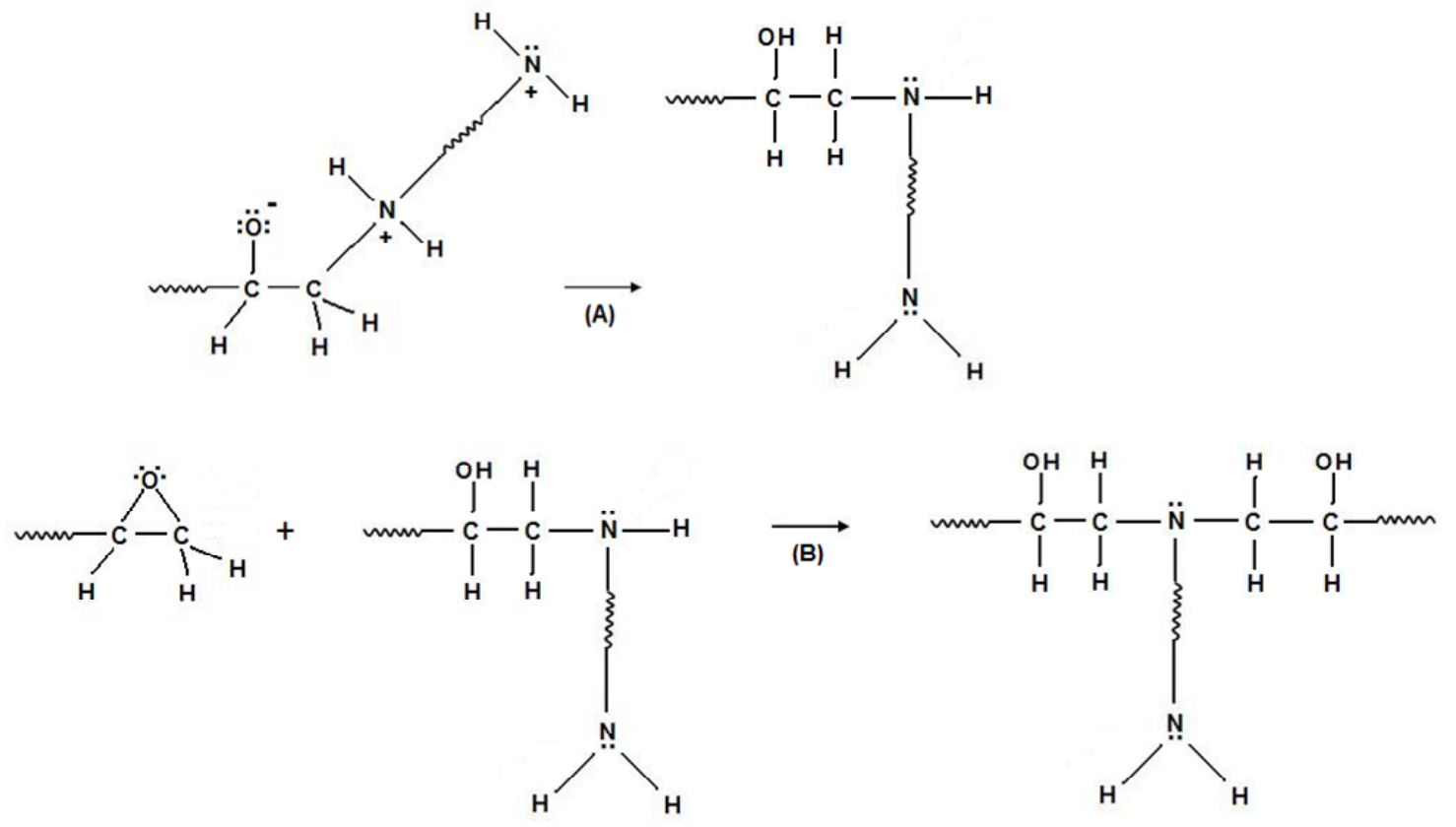

Figure 4. Second and Final steps of crosslinking reaction: (A) The negatively charged oxygen abstracts a proton from the neighboring protonated amine, resulting in an alcohol group and an amine group and the crosslinking is complete. (B) The same crosslinked nitrogen reacts with another epoxide end of EPON 862 and forms two crosslinks. 


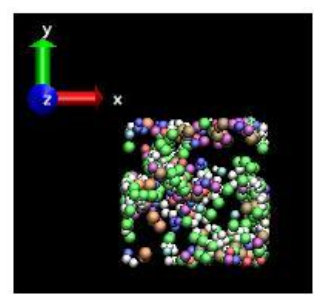

$16: 8$ crosslinked structure

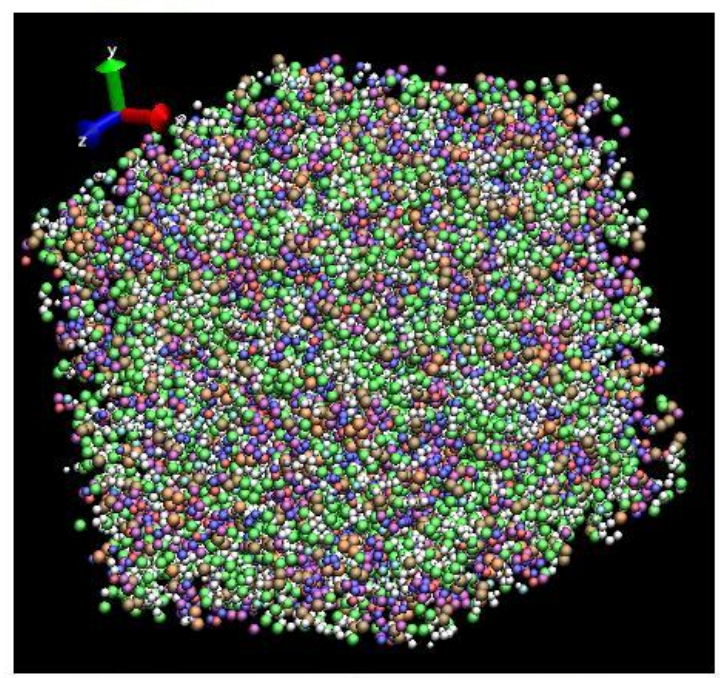

27 clusters shrunk and equilibrated to form a densely packed 432:216 structure at the right density

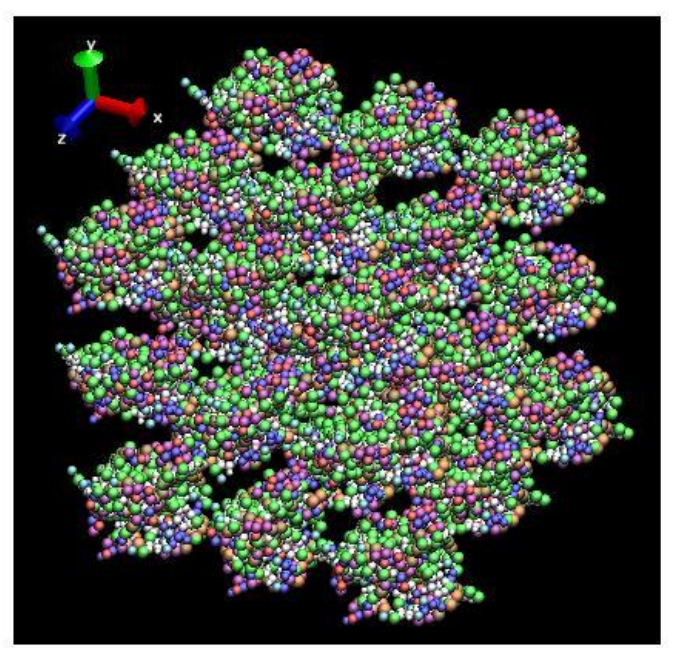

27 clusters arranged in a $3 \times 3 \times 3$ array and each cluster is a $16: 8$ crosslinked structure

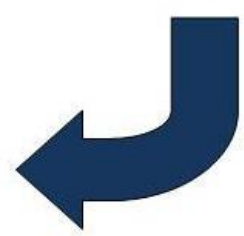

Figure 5. Evolution of EPON 862-DETDA model having 432:216 stoichiometric ratio. (Atom spheres are at the same resolution for comparison of sizes of simulation boxes in the 3 figures)

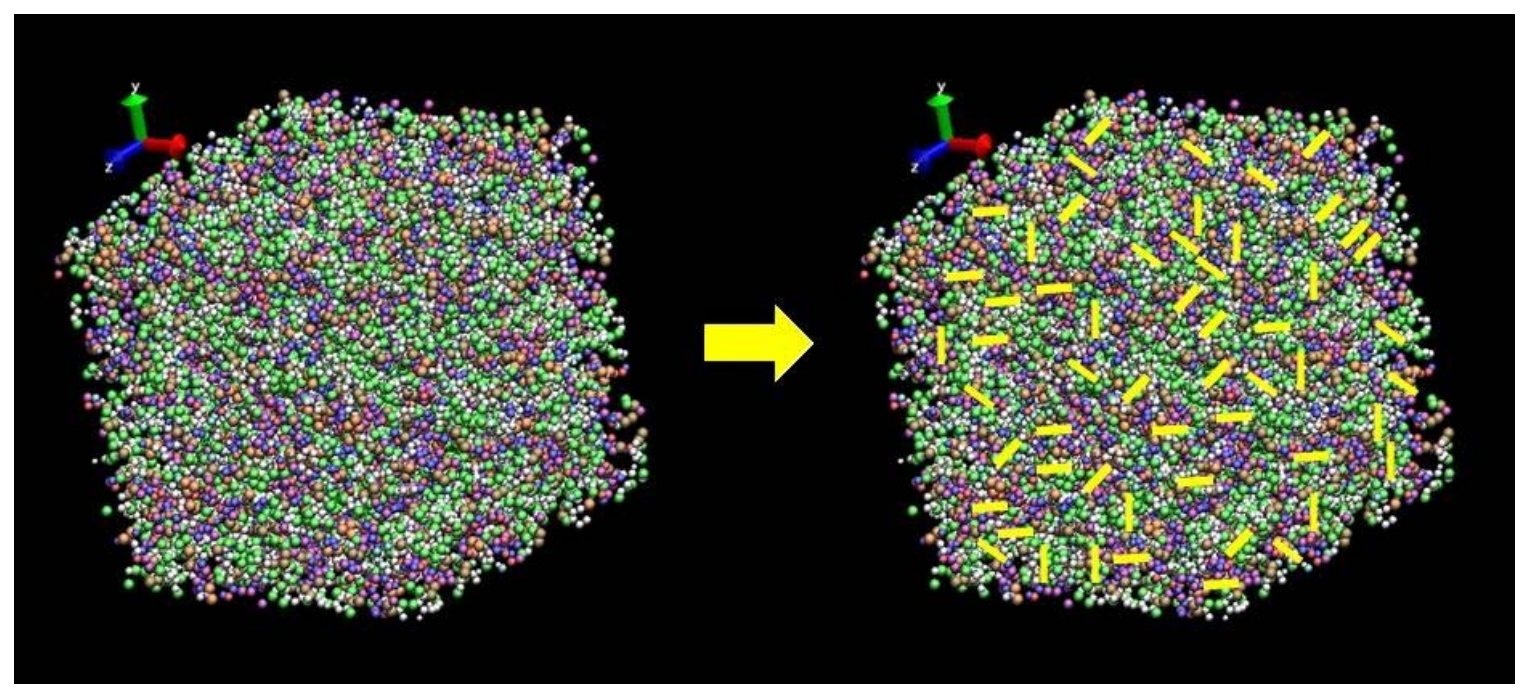

Figure 6. Crosslinking in between clusters of EPON 862-DETDA 432:216 model. (Yellow lines indicate crosslinked bonds connecting clusters) 


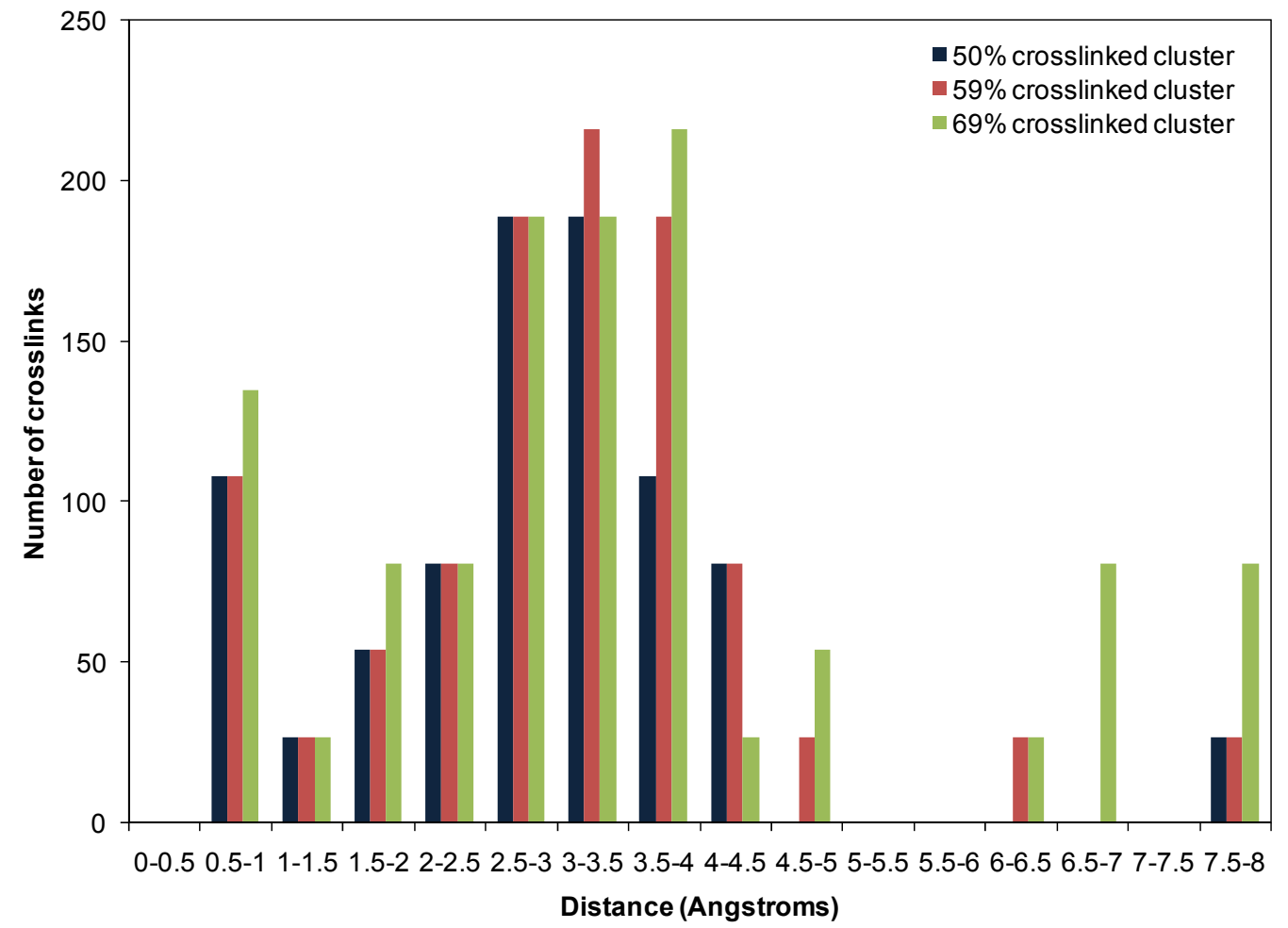

Figure 7. Frequency of crosslink formation over 0-8 Angstroms range within clusters

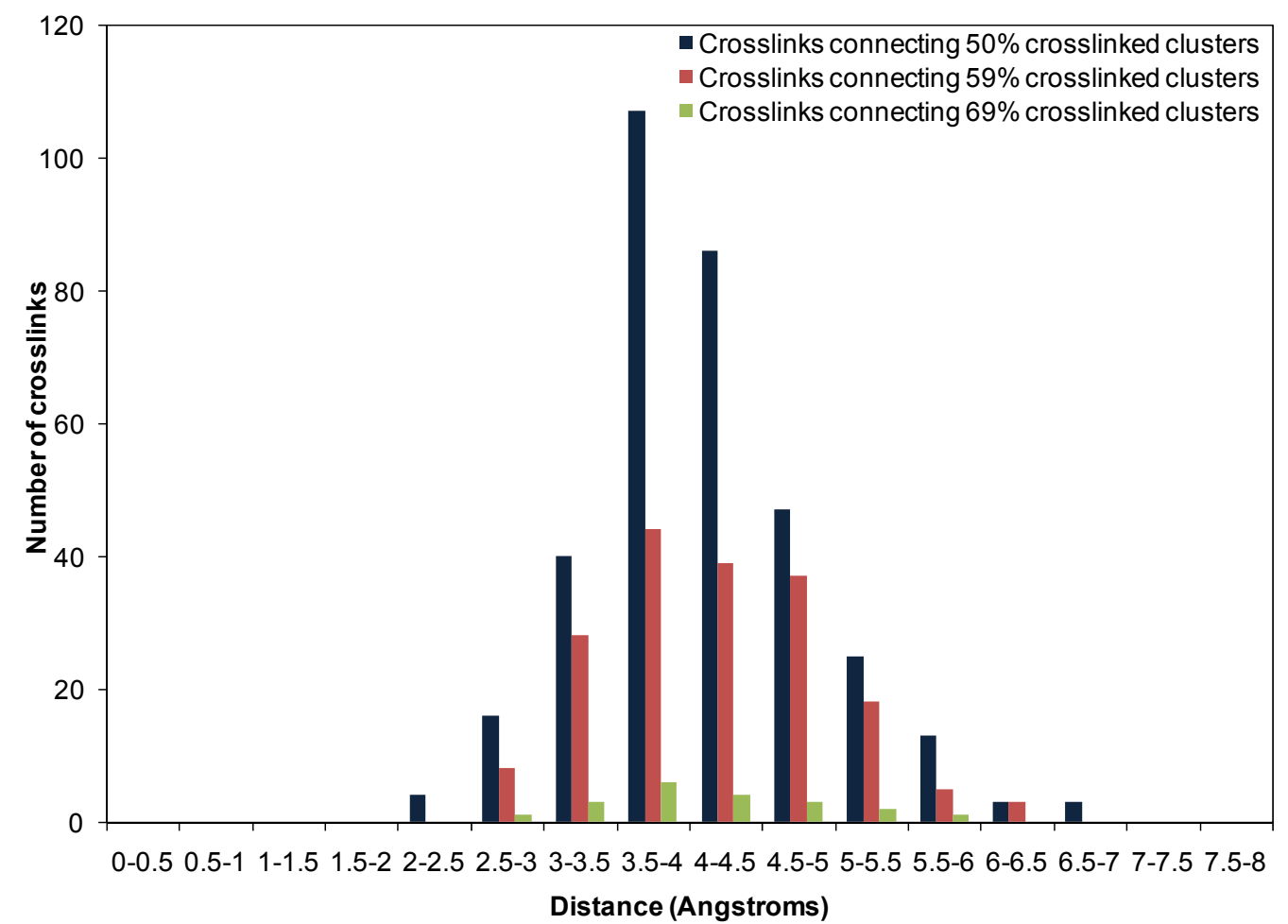

Figure 8. Frequency of crosslink formation for interconnecting clusters over $0-8$ Angstroms range for $70 \%$ crosslinked system. 


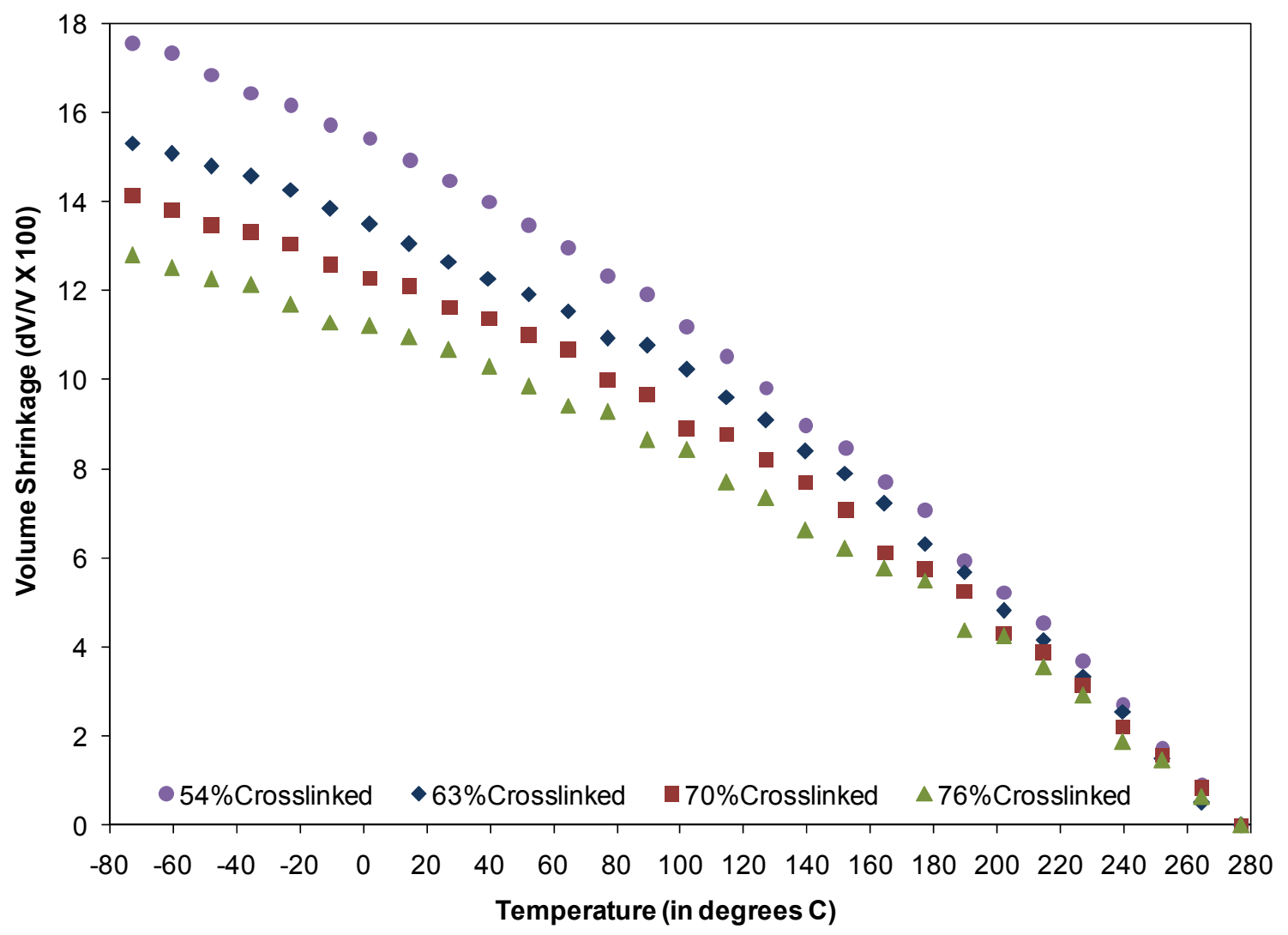

Figure 9. Volume shrinkage curves of $54 \%, 63 \%, 70 \%$ and $76 \%$ overall crosslinked systems with $50 \%$ crosslinked clusters with respect to the volume at $277^{\circ} \mathrm{C}$ 


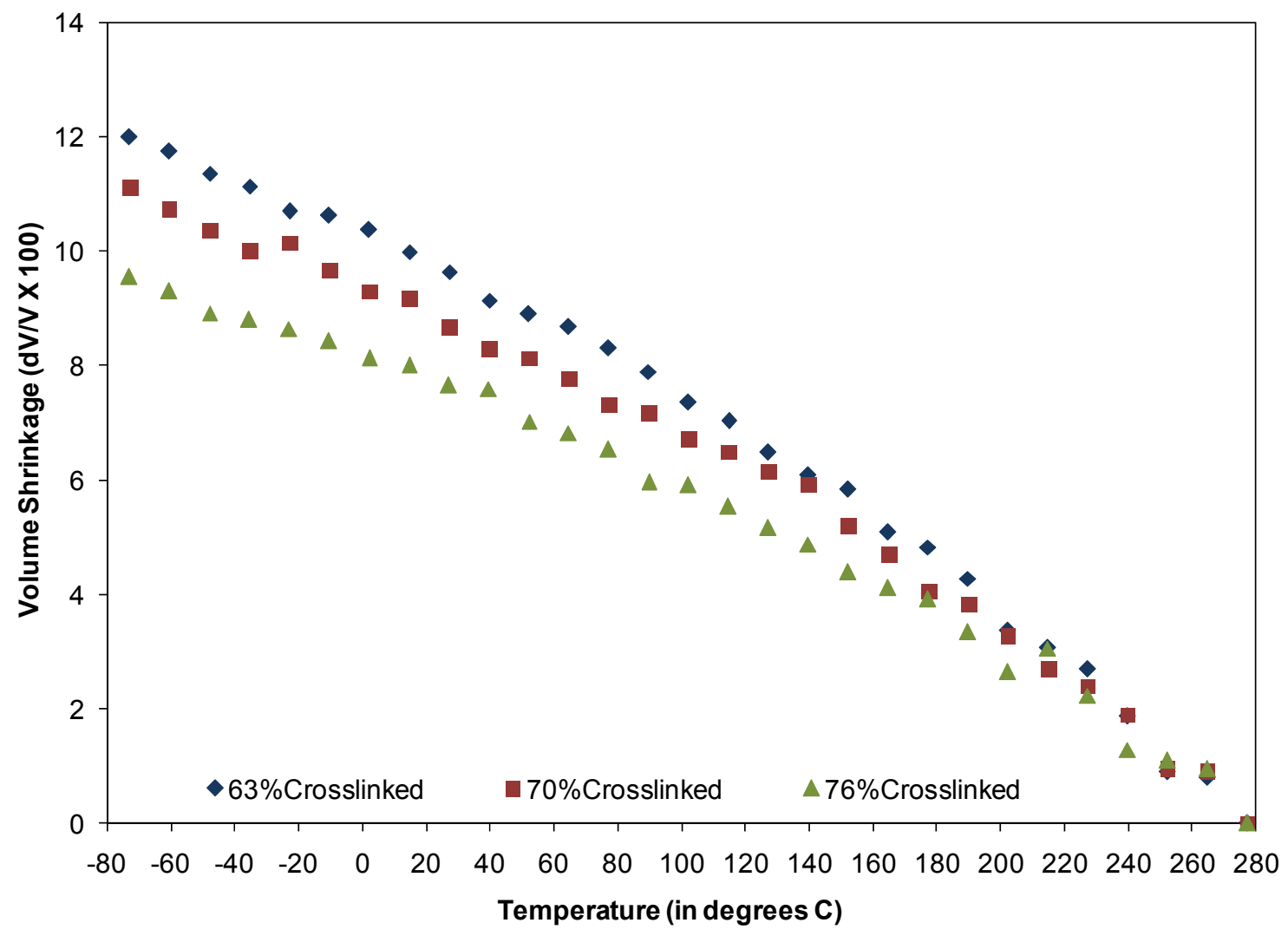

Figure 10 . Volume shrinkage curves of $63 \%, 70 \%$ and $76 \%$ overall crosslinked systems with $59 \%$ crosslinked clusters with respect to the volume at $277^{\circ} \mathrm{C}$ 


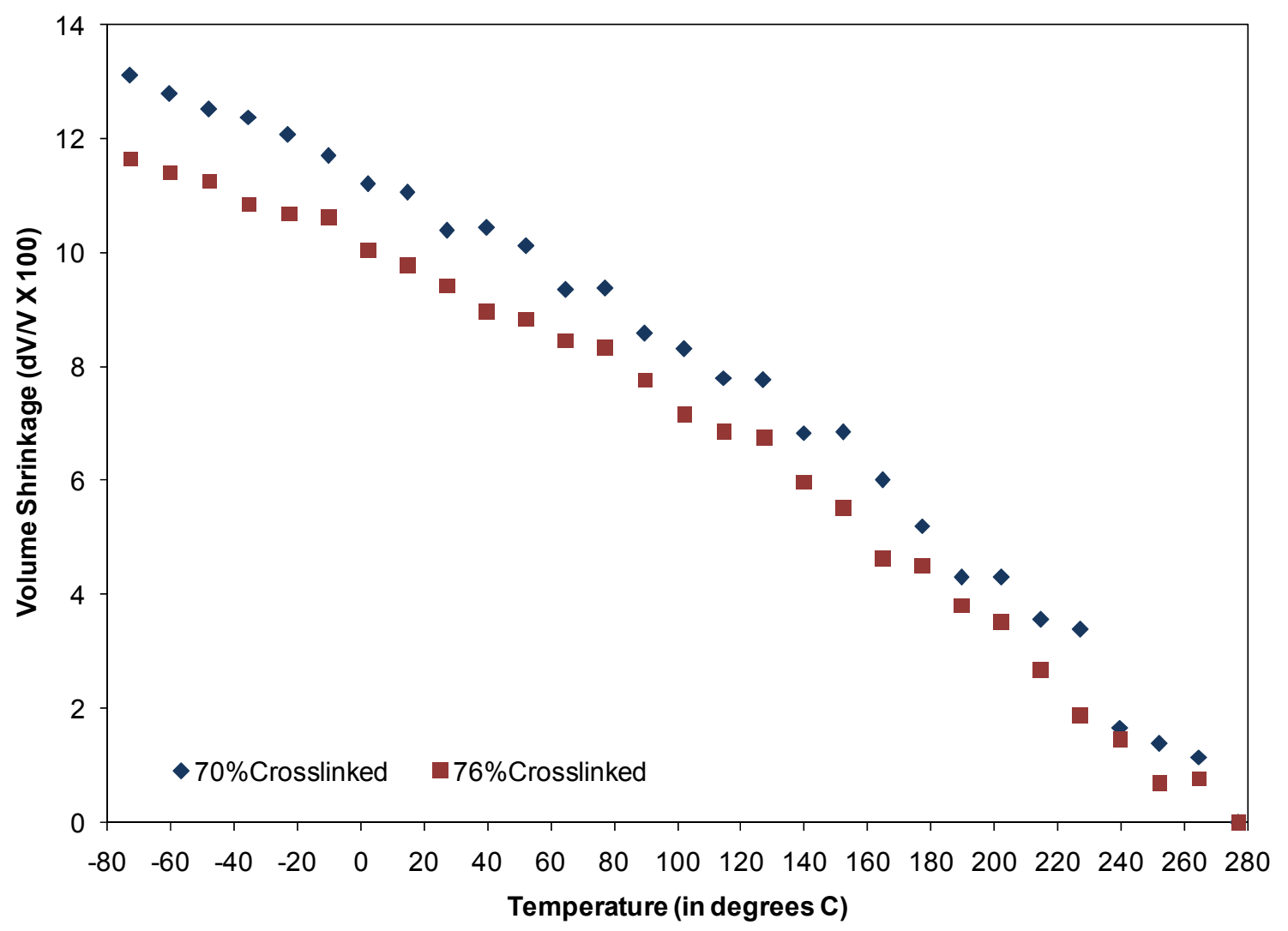

Figure 11. Volume shrinkage curves of $70 \%$ and $76 \%$ overall crosslinked systems with $69 \%$ crosslinked clusters with respect to the volume at $277^{\circ} \mathrm{C}$ 


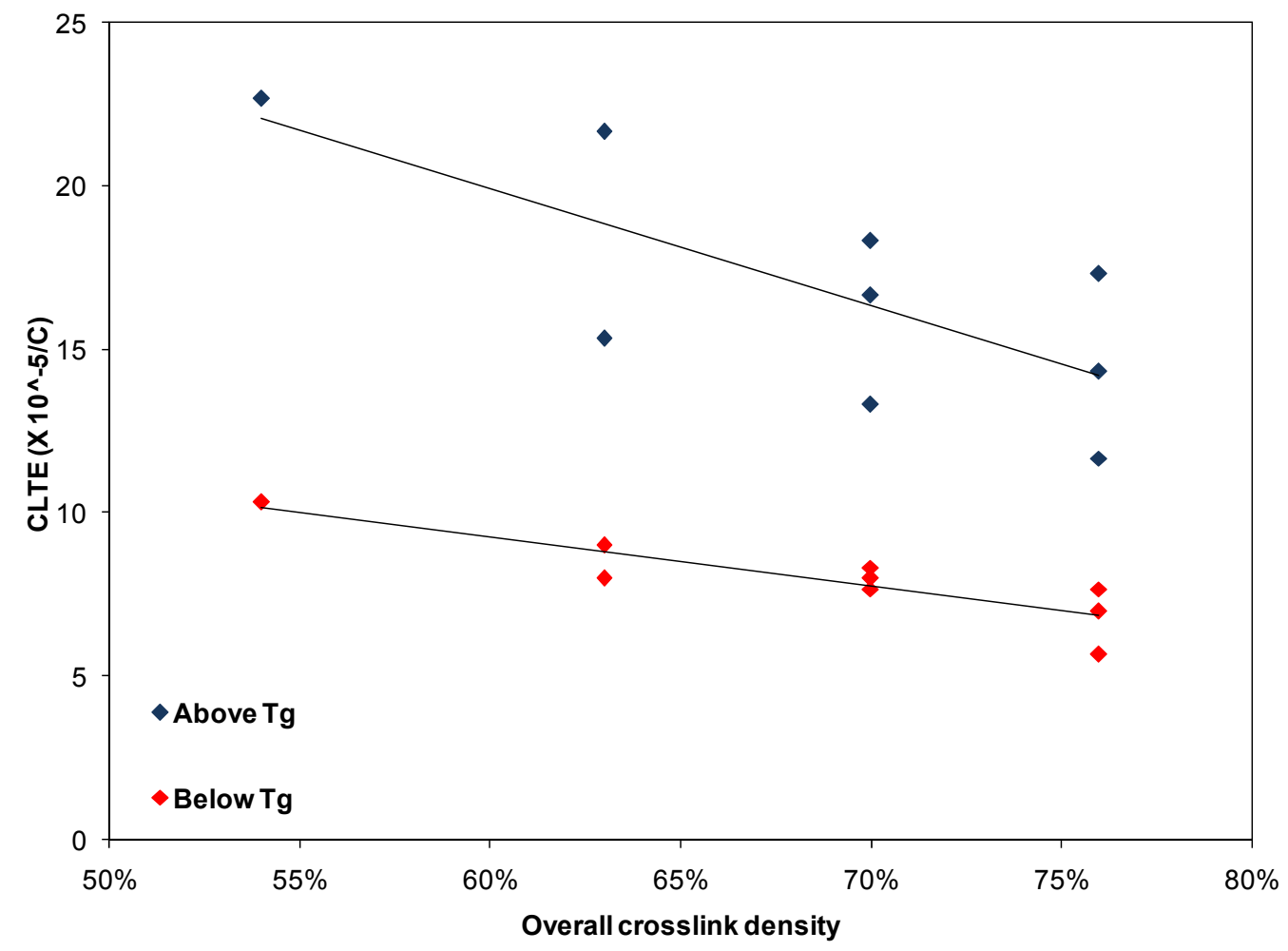

Figure 12. Coefficients of linear thermal expansion vs. overall crosslink density

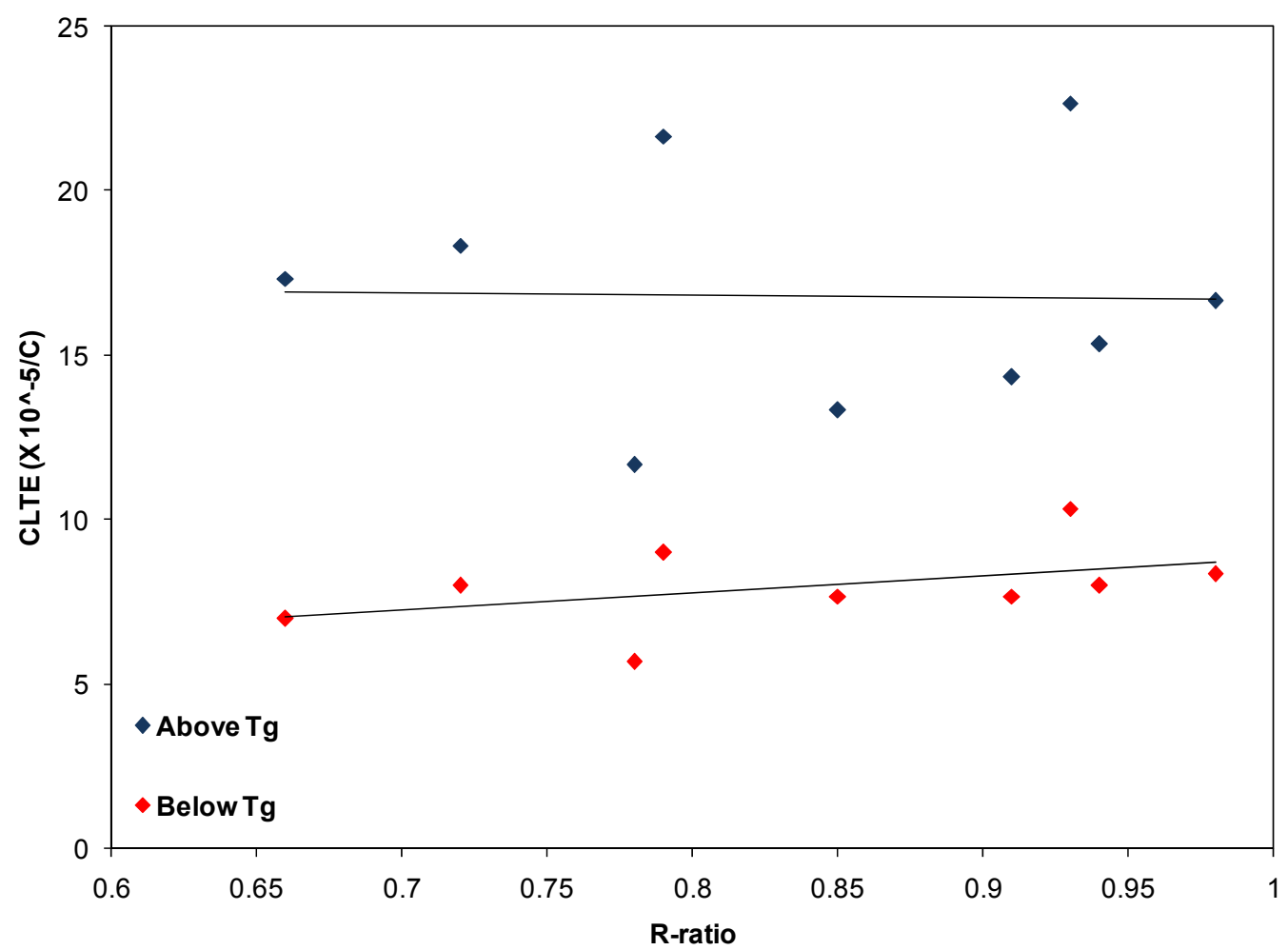

Figure 13. Coefficients of linear thermal expansion vs. R-ratio 


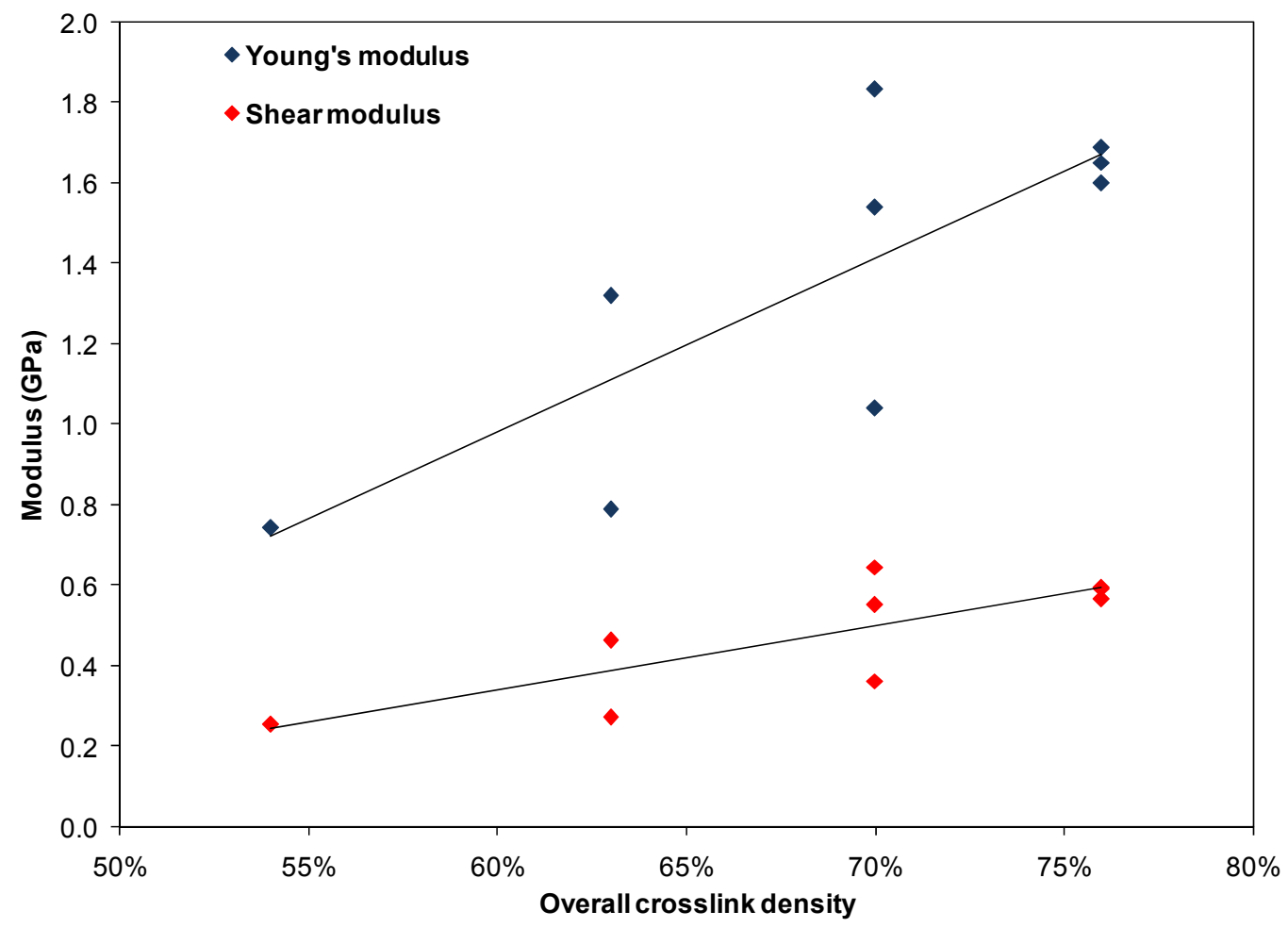

Figure 14. Young's and Shear moduli vs. overall crosslink density

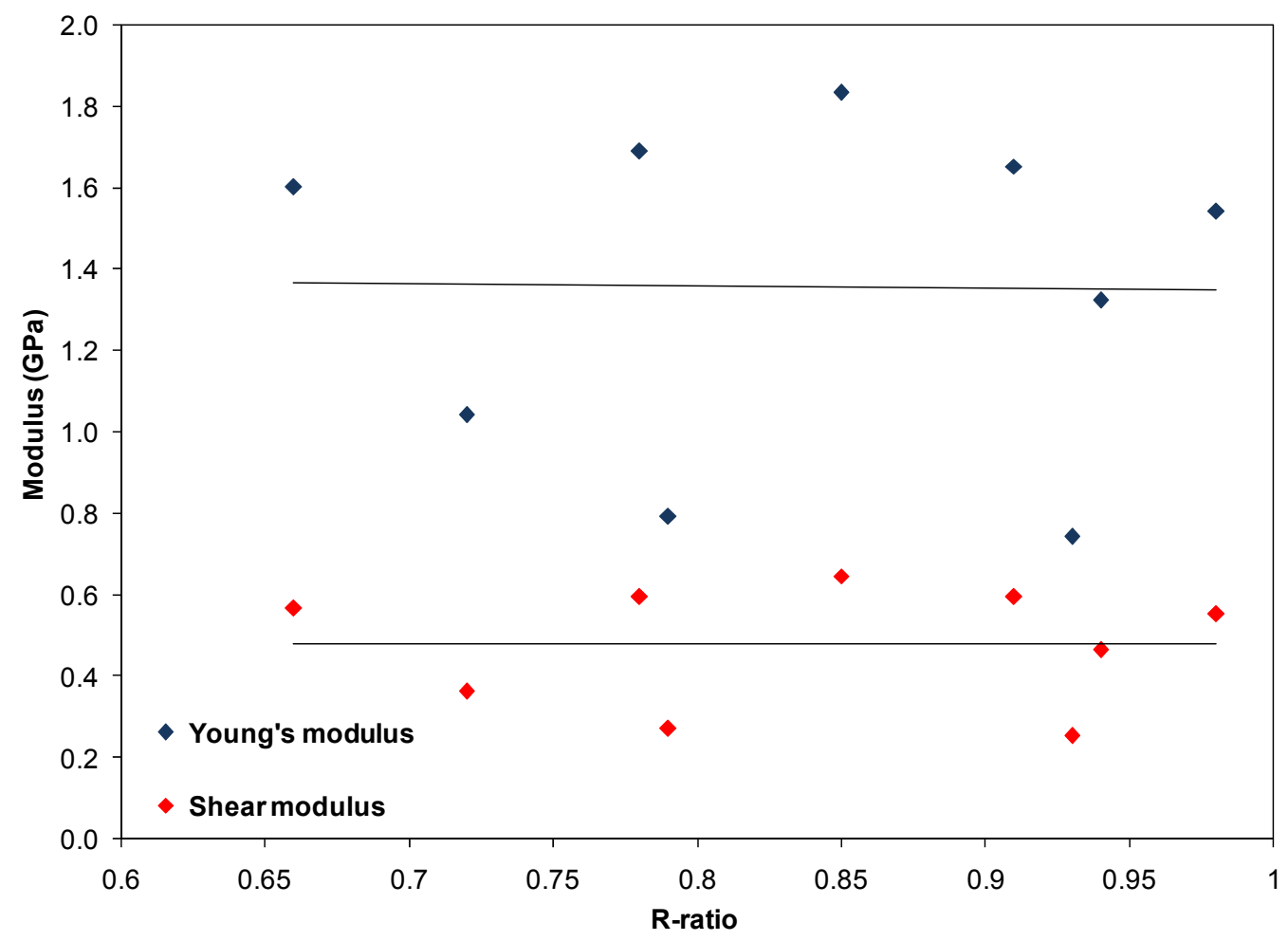

Figure 15. Young's and Shear moduli vs. R-ratio 